\title{
Nonperturbative evaluation of a field correlator appearing in the heavy quarkonium system
}

\author{
M. Baker \\ University of Washington, Seattle, WA 98105, USA \\ J. S. Ball \\ University of Utah, Salt Lake City, UT 84112, USA \\ N. Brambilla \\ Dipartimento di Fisica, Università di Milano, \\ INFN, Sezione di Milano, Via Celoria 16, 20133 Milano, Italy \\ A. Vairo \\ Dipartimento di Fisica, Università di Bologna, \\ Via Irnerio 46, 40126 Bologna, Italy
}

\begin{abstract}
Recently an electric and magnetic field correlator appearing in the description of the heavy quarkonium system was evaluated on the lattice. Here, we give a nonperturbative analytical evaluation of this field correlator using a dual description of long distance Yang-Mills theory and using the stochastic vacuum model. The two predictions are both compatible with lattice data but show a different dependence on the quark separation. We discuss the analytic results in relation to the lattice data.
\end{abstract}




\section{Introduction}

The semirelativistic interaction for a heavy quark-antiquark system is known in terms of electric and magnetic field correlators [1-7]. Due to confinement, these correlators cannot simply be evaluated in perturbation theory. However, it is possible to obtain them with lattice simulations. Analytic estimates of the correlator behaviour can be obtained by making some assumptions on the nature of the QCD vacuum or on the confinement mechanism. Until now the lattice results are compatible with, up to the available precision, the analytic calculations of the field correlators involved in the static and spin-dependent quark-antiquark semirelativistic interaction (see e.g. [4]). Recently, the correlators entering the velocity-dependent spin-independent part of the interaction were obtained on the lattice [8] and are compatible with the existing predictions (see $[3,5-7])$.

In [6] it was shown that a spin independent term, $\frac{1}{8}\left(\frac{1}{m_{1}^{2}}+\frac{1}{m_{2}^{2}}\right) \Delta V_{\mathrm{a}}$, arises at order $1 / \mathrm{m}^{2}$ in the quark-antiquark potential from the Darwin and the spinspin interaction. A previous analytic determination of $\Delta V_{\mathrm{a}}$ in the minimal area law model [6] gives zero. The first lattice QCD evaluation of $\Delta V_{\mathrm{a}}$ has just been carried out and gives non-zero negative value [8]. For quark-antiquark distances greater than $0.2 \sim 0.3 \mathrm{fm}$ its behaviour is compatible with a negative constant with a value between 0 and $-1.5 \mathrm{GeV}^{3}$. For distances shorter than $0.2 \mathrm{fm} \Delta V_{\mathrm{a}}$ falls off like $1 / r$.

In this letter we calculate $\Delta V_{\mathrm{a}}$ using a dual description of long distance YangMills theory [9]. We give also the predictions of the stochastic vacuum model [3] thereby completing a recently given comparison between these models [4]. Finally we compare the theoretical predictions with the lattice measurements and discuss the implication of these results for the confinement mechanism.

$\Delta V_{\mathrm{a}}$ is defined as following ${ }^{1}$ :

$$
\begin{aligned}
\Delta V_{\mathrm{a}} & =\Delta V_{\mathrm{a}}^{B}+\Delta V_{\mathrm{a}}^{E} \\
\Delta V_{\mathrm{a}}^{B} & =-\lim _{T \rightarrow \infty} \frac{i e^{2}}{T} \int_{-T / 2}^{T / 2} d t \int_{-T / 2}^{T / 2} d t^{\prime}\left\langle\left\langle\mathbf{B}\left(z_{1}\right) \cdot \mathbf{B}\left(z_{1}^{\prime}\right)\right\rangle\right\rangle-\left\langle\left\langle\mathbf{B}\left(z_{1}\right)\right\rangle\right\rangle \cdot\left\langle\left\langle\mathbf{B}\left(z_{1}^{\prime}\right)\right\rangle\right\rangle,
\end{aligned}
$$

$\overline{1}$ Here and in the following $e$ stands for the usual QCD coupling constant, $\alpha_{\mathrm{s}} \equiv$ $e^{2} / 4 \pi$, while $g$ denotes the dual coupling constant $g \equiv 2 \pi / e$. We have used this unconventional notation to make contact with the dual theory papers. 


$$
\Delta V_{\mathrm{a}}^{E}=\lim _{T \rightarrow \infty} \frac{i e^{2}}{T} \int_{-T / 2}^{T / 2} d t \int_{-T / 2}^{T / 2} d t^{\prime}\left\langle\left\langle\mathbf{E}\left(z_{1}\right) \cdot \mathbf{E}\left(z_{1}^{\prime}\right)\right\rangle\right\rangle-\left\langle\left\langle\mathbf{E}\left(z_{1}\right)\right\rangle\right\rangle \cdot\left\langle\left\langle\mathbf{E}\left(z_{1}^{\prime}\right)\right\rangle\right\rangle,
$$

where

$$
\begin{aligned}
E_{i}=F_{0 i}, \quad B_{i}=\hat{F}_{0 i}, \quad & F_{\mu \nu}=\partial_{\mu} A_{\nu}-\partial_{\nu} A_{\mu}+i e\left[A_{\mu}, A_{\nu}\right], \\
\hat{F}^{\mu \nu} & =\frac{1}{2} \epsilon^{\mu \nu \rho \sigma} F_{\rho \sigma},
\end{aligned}
$$

and

$$
\langle\langle f(A)\rangle\rangle=\frac{\int \mathcal{D} A e^{i S_{\mathrm{YM}}(A)} \operatorname{Tr} \mathrm{P}\left\{f(A) \exp \left[i e \oint_{\Gamma} d x^{\mu} A_{\mu}(x)\right]\right\}}{\int \mathcal{D} A e^{i S_{\mathrm{YM}}(A)} \operatorname{Tr} \mathrm{P} \exp \left[i e \oint_{\Gamma} d x^{\mu} A_{\mu}(x)\right]} .
$$

We define, also, the Wilson loop:

$$
W(\Gamma)=\mathrm{P} \exp \left[i e \oint_{\Gamma} d x^{\mu} A_{\mu}(x)\right] .
$$

The closed loop $\Gamma$ is defined by the quark (anti-quark) trajectories $\mathbf{z}_{1}(t)\left(\mathbf{z}_{2}(t)\right)$ where $t$ varies from the initial time $-T / 2$ to the final time $T / 2$. The quark (anti-quark) trajectories $\mathbf{z}_{1}(t)\left(\mathbf{z}_{2}(t)\right)$ define the world lines $\Gamma_{1}\left(\Gamma_{2}\right)$. The world lines $\Gamma_{1}$ and $\Gamma_{2}$, along with two straight-lines at fixed time connecting the end points, make up the contour $\Gamma$. We use also the notation $z_{1} \equiv z_{1}(t)$ and $z_{1}^{\prime} \equiv z_{1}\left(t^{\prime}\right)$. As usual $A_{\mu}(x) \equiv A_{\mu}^{a}(x) \lambda_{a} / 2$, Tr means the trace over colour indices, $\mathrm{P}$ prescribes the ordering of the colour matrices according to the direction fixed on the loop and $S_{\mathrm{YM}}(A)$ is the Yang Mills action including a gauge fixing term.

$\Delta V_{\mathrm{a}}$ is a gauge invariant quantity.

The field-strength expectation values in (2), (3) can be expressed as functional derivatives of $\log \langle W(\Gamma)\rangle$ with respect to the path $\Gamma$ [5]. In fact the change induced in $\langle W(\Gamma)\rangle$ by letting $z_{1}^{\mu}(t) \rightarrow z_{1}^{\mu}(t)+\delta z_{1}^{\mu}(t)$ where $\delta z_{1}^{\mu}(-T / 2)=$ $\delta z_{1}^{\mu}(T / 2)=0$ is

$$
\left\langle\left\langle F_{\mu \nu}\left(z_{1}\right)\right\rangle\right\rangle=\frac{\delta i \log \langle W(\Gamma)\rangle}{e \delta S^{\mu \nu}\left(z_{1}\right)},
$$




$$
\delta S^{\mu \nu}\left(z_{1}\right)=\left(d z_{1}^{\mu} \delta z_{1}^{\nu}-d z_{1}^{\nu} \delta z_{1}^{\mu}\right)
$$

and, varying the first quark line again, we have

$$
\left\langle\left\langle F_{\mu \nu}\left(z_{1}\right) F_{\lambda \rho}\left(z_{1}^{\prime}\right)\right\rangle\right\rangle-\left\langle\left\langle F_{\mu \nu}\left(z_{1}\right)\right\rangle\right\rangle\left\langle\left\langle F_{\lambda \rho}\left(z_{1}^{\prime}\right)\right\rangle\right\rangle=-\frac{\delta^{2} \log \langle W(\Gamma)\rangle}{e^{2} \delta S^{\mu \nu}\left(z_{1}\right) \delta S^{\lambda \rho}\left(z_{1}^{\prime}\right)} .
$$

Hence, the evaluation of (2) and (3) follows straightforwardly from the assumptions on the Wilson loop behaviour.

In the definition of $\Delta V_{\mathrm{a}}$ (eqs (2), (3)) the quarks are static, i. e. $\mathbf{z}_{1}$ and $\mathbf{z}_{2}$ are fixed in time. Accordingly the lattice calculations have been done inserting field-strength tensors in a rectangular Wilson loop.

In the static limit we note the following identities involving the static potential $[6]$ :

$$
\begin{gathered}
\int_{-T / 2}^{T / 2} d t \nabla V_{0}=e \int_{-T / 2}^{T / 2} d t\left\langle\left\langle\mathbf{E}\left(z_{1}\right)\right\rangle\right\rangle, \\
\int_{-T / 2}^{T / 2} d t \Delta V_{0}=-i e^{2} \int_{-T / 2}^{T / 2} d t \int_{-T / 2}^{T / 2} d t^{\prime}\left\langle\left\langle\mathbf{E}\left(z_{1}\right) \cdot \mathbf{E}\left(z_{1}^{\prime}\right)\right\rangle\right\rangle-\left\langle\left\langle\mathbf{E}\left(z_{1}\right)\right\rangle\right\rangle \cdot\left\langle\left\langle\mathbf{E}\left(z_{1}^{\prime}\right)\right\rangle\right\rangle \\
+e \int_{-T / 2}^{T / 2} d t\left\langle\left\langle\mathbf{D} \cdot \mathbf{E}\left(z_{1}\right)\right\rangle\right\rangle,
\end{gathered}
$$

where $D_{\mu}$ is the covariant derivative. Therefore, we have the following alternative way to express $\Delta V_{\mathrm{a}}^{B}$ and $\Delta V_{\mathrm{a}}^{E}$ :

$$
\begin{aligned}
\Delta V_{\mathrm{a}}^{B} & =\lim _{T \rightarrow \infty} \frac{i}{T} \int_{-T / 2}^{T / 2} d t \int_{-T / 2}^{T / 2} d t^{\prime} \frac{1}{4} \epsilon_{i l m} \epsilon_{i r s} \frac{\delta^{2} \log \langle W(\Gamma)\rangle}{\delta S_{l m}\left(z_{1}\right) \delta S_{r s}\left(z_{1}^{\prime}\right)}, \\
\Delta V_{\mathrm{a}}^{E} & =-\lim _{T \rightarrow \infty} \frac{i}{T} \int_{-T / 2}^{T / 2} d t \int_{-T / 2}^{T / 2} d t^{\prime} \frac{\delta^{2} \log \langle W(\Gamma)\rangle}{\delta S_{0 i}\left(z_{1}\right) \delta S_{0 i}\left(z_{1}^{\prime}\right)}, \\
& =-\int_{-T / 2}^{T / 2} d t \Delta V_{0}+e \int_{-T / 2}^{T / 2} d t\left\langle\left\langle\mathbf{D} \cdot \mathbf{E}\left(z_{1}\right)\right\rangle\right\rangle .
\end{aligned}
$$

The last term in (12) is the Darwin potential $[5,7]$. 


\section{$2 \quad$ Electric-magnetic duality}

We assume that for large loops the Wilson loop $W$ is the same as the Wilson loop $W_{\text {eff }}$ determined by an effective dual theory which is weakly coupled at long distances [7]:

$$
\langle W(\Gamma)\rangle=\left\langle W_{\text {eff }}(\Gamma)\right\rangle
$$

where

$$
\left\langle W_{\mathrm{eff}}(\Gamma)\right\rangle=\frac{\int \mathcal{D C} \mathcal{D} \mathcal{B} \exp \left[i \int d x \mathcal{L}_{\mathrm{eff}}\left(G_{\mu \nu}^{\mathrm{S}}\right)+\mathcal{L}_{\mathrm{GF}}\right]}{\int \mathcal{D C} \mathcal{D} \mathcal{B} \exp \left[i \int d x \mathcal{L}_{\mathrm{eff}}\left(G_{\mu \nu}^{\mathrm{S}}=0\right)+\mathcal{L}_{\mathrm{GF}}\right]}
$$

$\mathcal{L}_{\text {eff }}$ is the effective Lagrangian describing the interactions of dual potentials $\mathcal{C}_{\mu}$ and monopole fields $\mathcal{B}_{i}$; the dual potentials interact with quarks via the Dirac string tensor $G_{\mu \nu}^{\mathrm{S}}$ :

$$
G_{\mu \nu}^{\mathrm{S}}(x)=e \epsilon_{\mu \nu \alpha \beta} \int d s \int d \tau \frac{\partial y^{\alpha}}{\partial s} \frac{\partial y^{\beta}}{\partial \tau} \delta(x-y(s, \tau))
$$

where $y(s, \tau)$ is a world sheet with boundary $\Gamma$ swept out by the Dirac string. The colour electric field $D$ and the color magnetic field $H$ are the components of the dual field-strength tensor $\mathcal{G}_{\mu \nu}=\partial_{\mu} \mathcal{C}_{\nu}-\partial_{\nu} \mathcal{C}_{\mu}+G_{\mu \nu}^{\mathrm{S}}: D_{i}=\mathcal{G}_{0 i}$ and $H_{i}=\hat{\mathcal{G}}_{0 i}$. The Duality assumption (13) yields expressions for the Yang Mills expectation values (6) and (8) in terms of expectation values of the corresponding operators in the dual theory [7]

$$
\begin{aligned}
&\left\langle\left\langle\mathbf{B}\left(z_{1}\right)\right\rangle\right\rangle=\frac{4}{3}\left\langle\left\langle\mathbf{H}\left(z_{1}\right)\right\rangle\right\rangle_{\mathrm{eff}}, \\
&\left\langle\left\langle\mathbf{E}\left(z_{1}\right)\right\rangle\right\rangle=\frac{4}{3}\left\langle\left\langle\mathbf{D}\left(z_{1}\right)\right\rangle\right\rangle_{\mathrm{eff}}, \\
&\left\langle\left\langle\mathbf{B}\left(z_{1}\right) \cdot \mathbf{B}\left(z_{1}^{\prime}\right)\right\rangle\right\rangle-\left\langle\left\langle\mathbf{B}\left(z_{1}\right)\right\rangle\right\rangle \cdot\left\langle\left\langle\mathbf{B}\left(z_{1}^{\prime}\right)\right\rangle\right\rangle=-\frac{4}{3} i \frac{\delta\left\langle\left\langle H_{j}\left(z_{1}\right)\right\rangle\right\rangle_{\mathrm{eff}}}{\delta H_{j}^{\mathrm{S}}\left(z_{1}^{\prime}\right)}, \\
&\left\langle\left\langle\mathbf{E}\left(z_{1}\right) \cdot \mathbf{E}\left(z_{1}^{\prime}\right)\right\rangle\right\rangle-\left\langle\left\langle\mathbf{E}\left(z_{1}\right)\right\rangle\right\rangle \cdot\left\langle\left\langle\mathbf{E}\left(z_{1}^{\prime}\right)\right\rangle\right\rangle=-\frac{4}{3} i \frac{\delta\left\langle\left\langle D_{j}\left(z_{1}\right)\right\rangle\right\rangle_{\mathrm{eff}}}{\delta D_{j}^{\mathrm{S}}\left(z_{1}^{\prime}\right)},
\end{aligned}
$$

where $D_{i}^{\mathrm{S}}=G_{0 i}^{\mathrm{S}}, H_{i}^{\mathrm{S}}=\hat{G}_{0 i}^{\mathrm{S}}$. In the classical approximation to the dual theory (justified because the theory is weakly coupled at large distances) all quantities on the right-hand side of equations (14), (16)-(19) can be expressed in terms of the solutions of the classical equations of motion, in particular 


$$
\begin{aligned}
i \log \langle W(\Gamma)\rangle_{\mathrm{eff}} & =-\int d x \mathcal{L}_{\mathrm{eff}}\left(G_{\mu \nu}^{\mathrm{S}}\right) \\
\langle\langle\mathbf{D}(x)\rangle\rangle_{\mathrm{eff}} & =\mathbf{D}(\mathbf{x}) \\
\langle\langle\mathbf{H}(x)\rangle\rangle_{\mathrm{eff}} & =\mathbf{H}(\mathbf{x})
\end{aligned}
$$

The explicit evaluation of (18) in the classical configuration gives [7]:

$$
\Delta V_{\mathrm{a}}^{B}=-\left.\frac{4}{3} e^{2} \nabla_{\mathbf{x}} \cdot \nabla_{\mathbf{x}^{\prime}} G^{\mathrm{NP}}\left(\mathbf{x}, \mathbf{x}^{\prime}\right)\right|_{\mathbf{x}=\mathbf{x}^{\prime}=\mathbf{z}_{1}} .
$$

where the function $G^{\mathrm{NP}}$ satisfies the equation

$$
\left(-\Delta_{\mathbf{x}}+6 g^{2} \mathcal{B}^{2}(\mathbf{x})\right) G^{\mathrm{NP}}\left(\mathbf{x}, \mathbf{x}^{\prime}\right)=-\frac{6 g^{2} \mathcal{B}^{2}(\mathbf{x})}{4 \pi\left|\mathbf{x}-\mathbf{x}^{\prime}\right|}
$$

with $g=2 \pi / e$ and $\mathcal{B}$ is the static monopole field.

Now we use equation (24) to obtain the behaviour of $G^{\mathrm{NP}}$ near the quark sources. Let us introduce the vector

$$
\left.\mathbf{K}(\mathbf{x}) \equiv \nabla_{\mathbf{x}^{\prime}} G^{\mathrm{NP}}\left(\mathbf{x}, \mathbf{x}^{\prime}\right)\right|_{\mathbf{x}^{\prime}=\mathbf{z}_{1}}
$$

In terms of cylindrical coordinate with origin in $\mathbf{z}_{1}$, we have $\mathbf{x}-\mathbf{z}_{1}=z \mathbf{e}_{z}+\rho \mathbf{e}_{\rho}$, where the unit vector $\mathbf{e}_{z}$ is directed along $\mathbf{r}=\mathbf{z}_{1}-\mathbf{z}_{2}$ and the unit vector $\mathbf{e}_{\rho}$ is orthogonal to $\mathbf{e}_{z}$ in the plane of $\mathbf{x}$ and $\mathbf{r}$. By symmetry

$$
\mathbf{K}=K_{z} \mathbf{e}_{z}+K_{\rho} \mathbf{e}_{\rho}
$$

As a consequence, (24) can be splitted into two equations, one for each component:

$$
\begin{aligned}
& \left(-\Delta+6 g^{2} \mathcal{B}^{2}(\mathbf{x})\right) K_{z}=-\frac{6 g^{2} z \mathcal{B}^{2}(\mathbf{x})}{4 \pi\left|\mathbf{x}-\mathbf{z}_{1}\right|^{3}} \\
& \left(-\tilde{\Delta}+6 g^{2} \mathcal{B}^{2}(\mathbf{x})\right) K_{\rho}=-\frac{6 g^{2} \rho \mathcal{B}^{2}(\mathbf{x})}{4 \pi\left|\mathbf{x}-\mathbf{z}_{1}\right|^{3}}
\end{aligned}
$$

where, now, $\Delta=\frac{1}{\rho} \frac{\partial}{\partial \rho}+\frac{\partial^{2}}{\partial \rho^{2}}+\frac{1}{\rho^{2}} \frac{\partial^{2}}{\partial \phi^{2}}+\frac{\partial^{2}}{\partial z^{2}}$ and $\tilde{\Delta} \equiv \Delta-\frac{1}{\rho^{2}}$. Let $\theta$ be the angle between $\mathbf{r}$ and $\mathbf{x}-\mathbf{z}_{1}$, where for $\theta=\pi$ the vector $\mathbf{x}-\mathbf{z}_{1}$ is along $\mathbf{r}$, so that $z=\left|\mathbf{x}-\mathbf{z}_{1}\right| \cos \theta, \rho=\left|\mathbf{x}-\mathbf{z}_{1}\right| \sin \theta$. On the string, $\mathcal{B}$ must vanish, and its behaviour near quark 1 is given by [10]

$$
\mathcal{B}=b \sqrt{1+\cos \theta}\left|\mathbf{x}-\mathbf{z}_{1}\right|^{(\sqrt{3}-1) / 2},
$$


where $b$ is a function weakly dependent on $r$ (the quark-antiquark distance). In Table 1 we list values of $b$ obtained by numerical integration of the static equations for $\mathcal{B}$ for varying separations $r$. Putting (28) in (26)-(27) and keeping only terms which are singular near the quark 1 (for $\mathbf{x} \rightarrow \mathbf{z}_{1}$ ), we obtain

$$
\begin{aligned}
-\Delta K_{z} & =-\frac{3 g^{2} b^{2}}{2 \pi} \cos \theta(1+\cos \theta)\left|\mathbf{x}-\mathbf{z}_{1}\right|^{\sqrt{3}-3} \\
-\tilde{\Delta} K_{\rho} & =-\frac{3 g^{2} b^{2}}{2 \pi} \sin \theta(1+\cos \theta)\left|\mathbf{x}-\mathbf{z}_{1}\right|^{\sqrt{3}-3}
\end{aligned}
$$

The solutions of these equations are [10]

$$
\begin{aligned}
& K_{z}=-\frac{3 g^{2} b^{2}}{2 \pi}\left(-\frac{1}{3}-\frac{\cos \theta}{1-\sqrt{3}}+\frac{\cos ^{2} \theta}{3+\sqrt{3}}\right)\left|\mathbf{x}-\mathbf{z}_{1}\right|^{\sqrt{3}-1}, \\
& K_{\rho}=\frac{3 g^{2} b^{2}}{2 \pi}\left(\frac{1}{1-\sqrt{3}}-\frac{\cos \theta}{3+\sqrt{3}}\right) \sin \theta\left|\mathbf{x}-\mathbf{z}_{1}\right|^{\sqrt{3}-1} .
\end{aligned}
$$

Finally

$$
\begin{aligned}
\Delta V_{\mathrm{a}}^{B} & =-\left.\frac{4}{3} e^{2} \nabla_{\mathbf{x}} \cdot \nabla_{\mathbf{x}^{\prime}} G^{\mathrm{NP}}\left(\mathbf{x}, \mathbf{x}^{\prime}\right)\right|_{\mathbf{x}=\mathbf{x}^{\prime}=\mathbf{z}_{1}}=\left.\frac{4}{3} e^{2} \nabla_{\mathbf{x}} \cdot \mathbf{K}(\mathbf{x})\right|_{\mathbf{x}=\mathbf{z}_{1}} \\
& =-\left.8 \pi b^{2}(\sqrt{3}+2)\left|\mathbf{x}-\mathbf{z}_{1}\right|^{\sqrt{3}-2}\right|_{\mathbf{x}=\mathbf{z}_{1}} .
\end{aligned}
$$

The result (33) is divergent. However the dual theory has a natural ultraviolet cut-off which is the mass $M \approx 0.640 \mathrm{GeV}$ of the dual gluon, and we can put

$$
\left.\left|\mathbf{x}-\mathbf{z}_{1}\right|^{\sqrt{3}-2}\right|_{\mathbf{x}=\mathbf{z}_{1}}=M^{2-\sqrt{3}}
$$

From this prescription and the data of Table 1, we can derive the values of $\Delta V_{\mathrm{a}}^{B}$ at different distances as given in Table 2 . We observe that in the range of interest $(0.2 \mathrm{fm}<r<1 \mathrm{fm})$ the magnetic contribution to $\Delta V_{\mathrm{a}}$ can be considered almost a constant $\left(\approx-0.3 \mathrm{GeV}^{3}\right)$.

In order to evaluate $\Delta V_{\mathrm{a}}^{E}$ in the dual theory we use the following analogue of eq. (10) in the dual theory:

$$
\lim _{T \rightarrow \infty} \frac{1}{T} \int_{-T / 2}^{T / 2} d t \int_{-T / 2}^{T / 2} d t^{\prime} \frac{4}{3} e^{2} \frac{\delta\left\langle\left\langle D_{j}\left(z_{1}\right)\right\rangle\right\rangle_{\mathrm{eff}}}{\delta D_{j}^{\mathrm{S}}\left(z_{1}^{\prime}\right)}=
$$




$$
-\int_{-T / 2}^{T / 2} d t \Delta V_{0}+\frac{4}{3} e \int_{-T / 2}^{T / 2} d t \nabla \cdot\left\langle\left\langle\mathbf{D}\left(z_{1}\right)\right\rangle\right\rangle_{\mathrm{eff}} .
$$

Comparing (35) with (12) we obtain

$$
\int_{-T / 2}^{T / 2} d t\left\langle\left\langle\mathbf{D} \cdot \mathbf{E}\left(z_{1}\right)\right\rangle\right\rangle=\frac{4}{3} \int_{-T / 2}^{T / 2} d t \nabla \cdot\left\langle\left\langle\mathbf{D}\left(z_{1}\right)\right\rangle\right\rangle_{\mathrm{eff}} .
$$

Eq. (36) expresses the Darwin potential in terms of quantities calculated in the dual theory and has a simple interpretation. The left hand side is the expectation value of the electric current operator in Yang Mills theory and the right hand side is the corresponding quantity in the dual theory (note that (36) and (17) are independent results valid only on the quark trajectory). Since in the dual theory the colour electric field $\mathbf{D}$ is given by $\mathbf{D}=\nabla \times \mathbf{C}+\mathbf{D}^{S}$ the dual potential does not contribute to divergence of $\mathbf{D}$ and we have

$$
\nabla \cdot\left\langle\left\langle\mathbf{D}\left(z_{1}\right)\right\rangle\right\rangle_{\mathrm{eff}}=\nabla \cdot \mathbf{D}\left(z_{1}\right)=e \delta(\mathbf{r})
$$

where $\mathbf{r}=\mathbf{z}_{1}-\mathbf{z}_{2}$. The dynamics of the dual theory fixes $\nabla \times \mathbf{D}$, that is, the monopole current which, because of (36), does not contribute to the Darwin term. Thus the monopole degrees of freedom, which in the classical approximation to the dual theory produce the dual Meissner effect and confinement, contribute to $\Delta V_{\mathrm{a}}$ only via the first term on the right hand side of eq.(35). Thus comparison of $\Delta V_{\text {a }}$ predictions with lattice data provides a crucial test of the dual description of long distance Yang Mills theory.

Putting (37) in (35) we obtain

$$
\Delta V_{\mathrm{a}}^{E}=-\Delta V_{0}+\frac{16 \pi}{3} \alpha_{\mathrm{s}} \delta(\mathbf{r})=-\Delta\left(V_{0}+\frac{4}{3} \frac{\alpha_{\mathrm{s}}}{r}\right)
$$

where $\alpha_{\mathrm{s}}=e^{2} / 4 \pi$. The quantity $G^{\mathrm{NP}}$ determining $\Delta V_{\mathrm{a}}^{B}(33)$ is just the nonperturbative part of the monopole potential and so (33) is the natural magnetic counterpart of (38). Thus both the electric and magnetic parts of $\Delta V_{\mathrm{a}}$ directly measure the characteristic feature of the dual picture. The static potential $V_{0}$ obtained by solving numerically the equations of motion can be represented by the following analytic parametrization [11]:

$$
V_{0}(r)=-\frac{4}{3} \frac{\alpha_{\mathrm{s}}}{r} e^{-0.511 \sqrt{\sigma / \alpha_{\mathrm{s}}} r}+\sigma r-0.646 \sqrt{\sigma \alpha_{\mathrm{s}}}
$$


where $\sigma \approx 0.2 \mathrm{GeV}^{2}$ is the string tension. Substituting (39) in (38) we obtain

$$
\Delta V_{\mathrm{a}}^{E}=\frac{4}{3}(0.511)^{2} \frac{\sigma}{r} e^{-0.511 \sqrt{\sigma / \alpha_{\mathrm{s}}} r}-2 \frac{\sigma}{r} .
$$

In Table 3 we list some values of $\Delta V_{\mathrm{a}}^{E}$ for different quark-antiquark distances. Notice that the dependence on $r$ is strong. In particular the short-range behaviour $(r \rightarrow 0)$ is like $1 / r$. In Table 4 we sum up the magnetic and electric contributions and give the complete $\Delta V_{\mathrm{a}}$ behaviour. In the long-range region $(r \rightarrow \infty) \Delta V_{\mathrm{a}}$ has only magnetic contributions with an asymptotic value of $0.2 \sim 0.3 \mathrm{GeV}^{3}$. In the short-range region $\Delta V_{\mathrm{a}}$ has a behaviour like $1 / r$.

\section{Stochastic vacuum model}

In the stochastic vacuum model, one assumes that, in Euclidean space, the Wilson loop has the following behaviour [3]:

$$
\begin{aligned}
& \log \langle W(\Gamma)\rangle= \\
& \quad-\frac{1}{2} \int_{S} d S_{\mu \nu}(u) \int_{S} d S_{\lambda \rho}(v)\left(\delta_{\mu \lambda} \delta_{\nu \rho}-\delta_{\mu \rho} \delta_{\nu \lambda}\right)\left(D\left(h^{2}\right)+D_{1}\left(h^{2}\right)\right) \\
& \quad+\left(h_{\mu} h_{\lambda} \delta_{\nu \rho}-h_{\mu} h_{\rho} \delta_{\nu \lambda}+h_{\nu} h_{\rho} \delta_{\mu \lambda}-h_{\nu} h_{\lambda} \delta_{\mu \rho}\right) \frac{\partial}{\partial h^{2}} D_{1}\left(h^{2}\right),
\end{aligned}
$$

where $h \equiv u-v$. The functions $D$ and $D_{1}$, called correlator functions, can be interpreted as vacuum form factors. The perturbative contributions (contained in $D_{1}$ ) can be evaluated by the usual $e^{2}$ expansion of the Wilson loop, while the nonperturbative contributions, dominant in the long-range behaviour come only from lattice calculations [12]. The integration is performed over the minimal area surface $S$ with contour $\Gamma$. Up to order $1 / m^{2}$ the minimal surface can be identified exactly with the surface spanned by the straight-line joining the point $\left(t, \mathbf{z}_{1}(t)\right)$ on the quark line 1 with the point $\left(t, \mathbf{z}_{2}(t)\right)$ on the quark line 2 of $\Gamma$. Therefore, for our purposes, the generic point $u^{\mu}(t, s)$ on the surface $S$ will be given by:

$$
u^{0}=t \quad \mathbf{u}=s \mathbf{z}_{1}(t)+(1-s) \mathbf{z}_{2}(t)
$$

and the surface element $d S_{\mu \nu}(u)$ by

$$
\begin{aligned}
d S_{4 j}(u) & =d t d s r_{j}(t) \\
d S_{i j}(u) & =d t d s\left(s \dot{z}_{1 i}(t)+(1-s) \dot{z}_{2 i}(t)\right) r_{j}(t)
\end{aligned}
$$


where $t \in[-T / 2, T / 2], s \in[0,1]$ and $\mathbf{r}=\mathbf{z}_{1}-\mathbf{z}_{2}$.

From equation (8) and (41), expanding

$$
z_{1}\left(t^{\prime}\right)=z_{1}(t)+\left(t-t^{\prime}\right) \dot{z}_{1}(t)+\cdots
$$

we have in the static limit (i. e. neglecting the velocity dependent terms)

$$
\frac{\delta \log \langle W(\Gamma)\rangle}{\delta S_{l m}\left(z_{1}\right) \delta S_{r s}\left(z_{1}^{\prime}\right)}=-\left(\delta_{l r} \delta_{m s}-\delta_{l s} \delta_{m r}\right)\left(D\left(t^{2}\right)+D_{1}\left(t^{2}\right)\right) .
$$

Putting this expression in (11) we obtain

$$
\Delta V_{\mathrm{a}}^{B}=-6 \int_{0}^{\infty} d t\left(D\left(t^{2}\right)+D_{1}\left(t^{2}\right)\right)
$$

In an analogous way, since in the static limit

$$
\frac{\delta \log \langle W(\Gamma)\rangle}{\delta S_{0 i}\left(z_{1}\right) \delta S_{0 i}\left(z_{1}^{\prime}\right)}=-3\left(D\left(t^{2}\right)+D_{1}\left(t^{2}\right)+t^{2} \frac{d}{d t^{2}} D_{1}\left(t^{2}\right)\right)
$$

we obtain, from the first equality in equation (12)

$$
\Delta V_{\mathrm{a}}^{E}=-6 \int_{0}^{\infty} d t\left(D\left(t^{2}\right)+\frac{1}{2} D_{1}\left(t^{2}\right)\right)
$$

where a partial integration was performed.

In the usual long-range parameterization, suggested by the direct lattice evaluation of the correlator functions $D$ and $D_{1}[4,12]$,

$$
\begin{aligned}
& D\left(x^{2}\right)=d e^{-\delta|x|}, \quad \delta \approx 1 \mathrm{GeV}, \quad d \approx 0.073 \mathrm{GeV}^{4}, \\
& D_{1}\left(x^{2}\right)=d_{1} e^{-\delta_{1}|x|}, \quad \delta_{1} \approx 1 \mathrm{GeV}, \quad d_{1} \approx 0.0254 \mathrm{GeV}^{4} \text {, }
\end{aligned}
$$

the electric and magnetic contribution to $\Delta V_{\mathrm{a}}$ are

$$
\begin{aligned}
& \Delta V_{\mathrm{a}}^{B}=-6 \frac{d}{\delta}-6 \frac{d_{1}}{\delta_{1}} \approx-0.59 \mathrm{GeV}^{3}, \\
& \Delta V_{\mathrm{a}}^{E}=-6 \frac{d}{\delta}-3 \frac{d_{1}}{\delta_{1}} \approx-0.51 \mathrm{GeV}^{3},
\end{aligned}
$$


and then

$$
\Delta V_{\mathrm{a}}=\Delta V_{\mathrm{a}}^{B}+\Delta V_{\mathrm{a}}^{E} \approx-1.1 \mathrm{GeV}^{3}
$$

In contrast with the dual theory the long range contributions to $\Delta V_{\mathrm{a}}$ in the stochastic vacuum model are self energy corrections independent of $r$. For this reason these terms were neglected in [4] and did not appear in the minimal area law evaluation of [6]. We expect that a $r$ dependence will arise in the short range region, taking into account higher order perturbative contributions. Since the $r$ dependence is very weak also in the dual theory for the magnetic contribution $\Delta V_{\mathrm{a}}^{B}$, the difference between the two models is in the electric contributions $\Delta V_{\mathrm{a}}^{E}$ and is mainly due to the Darwin term. In fact, in the static limit the dual theory predicts

$$
e \int_{-T / 2}^{T / 2} d t\left\langle\left\langle\mathbf{D} \cdot \mathbf{E}\left(z_{1}\right)\right\rangle\right\rangle=\frac{4}{3} e^{2} \int_{-T / 2}^{T / 2} d t \delta(\mathbf{r})
$$

Eq.(55) reflects the fact that in the dual theory the color electric field is generated by the monopole current which does not contribute to the right hand side of eq.(36). This contrasts with the corresponding result of the stochastic vacuum model:

$$
e \int_{-T / 2}^{T / 2} d t\left\langle\left\langle\mathbf{D} \cdot \mathbf{E}\left(z_{1}\right)\right\rangle\right\rangle=\int_{-T / 2}^{T / 2} d t \nabla V_{0}+\text { self-energy contributions. }
$$

\section{Conclusion}

The dual theory predictions of $\Delta V_{\mathrm{a}}$ are in very good agreement with the lattice data. The asymptotic value for large distances is negative and very well inside the error bars of the lattice prediction. Surprisingly also the short range prediction, which in principle could be beyond the validity range of the dual theory, reproduces the $1 / r$ behaviour of the lattice fit. Also the long range result of the stochastic vacuum model is negative and compatible with the lattice data, but of self-energy type. A $r$ dependence arises from the perturbative contributions only at the next-to-leading order. However the main contribution to the $\frac{1}{r}$ behavior of $V_{\mathrm{a}}^{E}$ in the dual theory comes from the second term on the right hand side of eq. (40), which is clearly nonperturbative. Furthermore, as is seen from Table $2, V_{\mathrm{a}}^{B}$ also increases with decreasing $r$. After this calculation the main difference in the two models seems to be clear. In the dual theory only the quark charges contribute in the static limit to the Darwin potential, while 
in the stochastic vacuum model the Darwin potential is related to the full static potential. This means that a truly accurate lattice determination of the field correlator (which is possible for the magnetic part of the correlator and it is already in progress [13]) is of great interest and could provide significant information concerning the nonperturbative quark interaction.

\section{References}

[1] K. G. Wilson, Phys. Rev. D 10 (1974) 2445; E. Eichten and F. Feinberg, Phys. Rev. D 23 (1981) 2724; see also e.g. E. Eichten, in "Lattice '90", eds. V. A. Heller et al., Nucl. Phys. B 20 (Proc. Suppl.) (1991) 475;

[2] In the context of heavy-quark effective theory see e.g. C. Davies in "Quark Confinement and the Hadron Spectrum II", eds. N. Brambilla and G. M. Prosperi, (World Scientific, Singapore, 1996);

[3] H. G. Dosch, Phys. Lett. B 190 (1987) 177; H. G. Dosch and Yu. A. Simonov, Phys. Lett. B 205 (1988) 339; Yu. A. Simonov, Nucl. Phys. B 307 (1988) 512; B 324 (1989) 67;

[4] N. Brambilla and A. Vairo, Heavy quarkonia: Wilson area Law, Stochastic Vacuum Model and Dual QCD, IFUM 533/FT (1996);

[5] N. Brambilla, P. Consoli and G. M. Prosperi, Phys. Rev. D 50 (1994) 5878; N. Brambilla and G. M. Prosperi, Wilson loops, $q \bar{q}$ and $3 q$ potentials, BetheSalpeter equation, in "Quark Confinement and the Hadron Spectrum", eds. N. Brambilla and G. M. Prosperi, (World Scientific, Singapore, 1995);

[6] A. Barchielli, E. Montaldi and G. M. Prosperi, Nucl. Phys. B 296 (1988) 625;

[7] M. Baker, J. S. Ball, N. Brambilla, G. M. Prosperi and F. Zachariasen, UW/PT 96-03 (1996) (Phys. Rev. D in press);

[8] G. Bali, in "Quark Confinement and the Hadron Spectrum II", eds. N. Brambilla and G. M. Prosperi, (World Scientific, Singapore, 1996);

[9] M. Baker, J. S. Ball and F. Zachariasen, Phys. Rep. 209 (1991) 73;

[10] M. Baker, J. S. Ball, F. Zachariasen, Phys. Rev. D 44 (1991) 3328; 3949;

[11] M. Baker, J. S. Ball and F. Zachariasen, Phys. Rev. D 51 (1995) 1968;

[12] M. Campostrini, A. Di Giacomo and G. Mussardo, Z. Phys. C 25 (1984) 173; A. Di Giacomo and H. Panagopoulos Phys. Lett. B 285 (1992) 133; A. Di Giacomo, E. Meggiolaro and H. Panagopoulos, (March 1996) hep-lat/9603017;

[13] G. Bali, private communications. 
Table 1

$b$ vs $r$, the $q \bar{q}$ distance. The table shows a slight dependence.

\begin{tabular}{|l|c|}
\hline$r(\mathrm{fm})$ & $b^{2} / M^{\sqrt{3}-1}\left(\mathrm{GeV}^{2}\right)$ \\
\hline 0.175 & 0.007 \\
0.26 & 0.006 \\
0.35 & 0.0055 \\
0.7 & 0.0048 \\
1.4 & 0.0045 \\
\hline
\end{tabular}

Table 2

$\Delta V_{\mathrm{a}}^{B}$ at the ultraviolet cut-off $M=0.640 \mathrm{Gev}$ vs $r$, the $q \bar{q}$ distance $\left(\alpha_{\mathrm{s}}=0.37\right.$ and $\left.\sigma=0.2 \mathrm{Gev}^{2}\right)$.

\begin{tabular}{|l|c|}
\hline$r(\mathrm{fm})$ & $\Delta V_{\mathrm{a}}^{B}\left(\mathrm{GeV}^{3}\right)$ \\
\hline 0.175 & -0.407 \\
0.26 & -0.350 \\
0.35 & -0.320 \\
0.7 & -0.277 \\
1.4 & -0.263 \\
\hline
\end{tabular}


Table 3

$\Delta V_{\mathrm{a}}^{E}$ vs $r$, the $q \bar{q}$ distance $\left(\alpha_{\mathrm{s}}=0.37\right.$ and $\left.\sigma=0.2 \mathrm{Gev}^{2}\right)$.

\begin{tabular}{|l|c|}
\hline$r(\mathrm{fm})$ & $\Delta V_{\mathrm{a}}^{E}\left(\mathrm{GeV}^{3}\right)$ \\
\hline 0.175 & -0.402 \\
0.26 & -0.275 \\
0.35 & -0.208 \\
0.7 & -0.109 \\
1.4 & -0.057 \\
\hline
\end{tabular}

Table 4

$\Delta V_{\mathrm{a}}=\Delta V_{\mathrm{a}}^{B}+\Delta V_{\mathrm{a}}^{E}$ vs $r$, the $q \bar{q}$ distance $\left(\alpha_{\mathrm{s}}=0.37\right.$ and $\left.\sigma=0.2 \mathrm{Gev}^{2}\right)$.

\begin{tabular}{|l|c|}
\hline$r(\mathrm{fm})$ & $\Delta V_{\mathrm{a}}\left(\mathrm{GeV}^{3}\right)$ \\
\hline 0.175 & -0.809 \\
0.26 & -0.625 \\
0.35 & -0.528 \\
0.7 & -0.386 \\
1.4 & -0.320 \\
\hline
\end{tabular}

